\title{
Antibody responses to tetanus and diphtheria vaccine in chronic and recurrent rhinosinusitis*
}

\author{
Olli Tahkokallio ${ }^{1}$, Anni Koskinen ${ }^{1}$, Erna Kentala ${ }^{1}$, Rose-Marie Ölander ${ }^{2}$, \\ Petri Severi Mattila ${ }^{1}$ \\ Department of Otorhinolaryngology, Helsinki University Central Hospital, Finland \\ 2 National Institute for Health and Welfare, Helsinki, Finland
}

\section{SUMMARY}

\begin{abstract}
Objectives: Chronic rhinosinusitis may be accompanied by impaired immunity despite normal levels of serum immunoglobulins. Immune responses in sinusitis patients have previously been evaluated using polysaccharide vaccines.

Our aim was to assess the immune status by evaluating responses to diphtheria and tetanus vaccine.

Methods: Specific antibodies were measured before and 2 weeks after vaccination in 25 patients with chronic or recurrent sinusitis and in 30 healthy individuals. The mean age of the patients was 46 years and that of healthy controls 43 years.

Results: After vaccination the patients had on average 4.08-fold lower responses to diphtheria toxoid and 2.20-fold lower responses to tetanus than the controls. Fourteen out of 25 patients had antibody levels that did not reach the 95\% normal distribution range of healthy controls after either diphtheria or tetanus vaccination. All the patients had normal levels of serum immunoglobulins.

Conclusions: A significant proportion of patients with persisting symptoms of rhinosinisitis may have impaired responses to protein vaccines. Responses to protein vaccines may be used to evaluate immune function of sinusitis patients.
\end{abstract}

Key words: sinusitis, vaccination, antibodies, immunoglobulins, immunodeficiency

\section{INTRODUCTION}

Sinusitis is a frequent symptom in profound disorders of antibody mediated immunity such as common variable immune immune deficiency (CVID) ${ }^{(1-3)}$ and hyper IgM syndrome ${ }^{(4,5)}$. This suggests that antibodies are important in the resolution of sinusitis. Thereby, it is not surprising that patients who initially present with chronic rhinosinusitis (CRS), that is defined by inflammation of the mucosal lining of the sinuses lasting more than 12 weeks ${ }^{(6)}$, include patients who have impairments in their antibody-mediated immunity ${ }^{(7)}$.

Strategies for the evaluation of immune function in patients with CRS comprise assessments of allergy, measurements of serum IgA, IgM, and IgG subclasses, determination of specific antibody responses to vaccines, and HIV testing ${ }^{(8)}$. As inhalant allergy is a frequent contributing factor, the initial immune evaluation of CRS patients includes allergy testing.

Determinations of serum immunoglogulin values are useful, as some CRS patients have abnormal levels. Isolated or combined IgG3, IgG2, or IgA deficiencies have been observed both in adult and pediatric patients with $\mathrm{CRS}^{(9-15)}$. Even when serum immunoglobulin levels are within normal limits, antibody mediated-immunity maybe somewhat impaired. An example of this is that, despite serum $\operatorname{IgA}$ levels are within normal limits, the average serum IgA levels may still be lower in sinusitis patients than in healthy age and sex matched individuals ${ }^{(16)}$. As some patients have impaired immunity despite normal immunoglobulin levels, vaccine responses have been measured to assess immune function. Impaired responses to pneumococcal or Haemophilus influenzae polysaccharide vaccines have been observed both in children and adults with $\mathrm{CRS}^{(17-20)}$.

We present here a series of patients, who have normal levels of serum immunoglobulins, but who have continuing symptoms of sinusitis despite sinus surgery and numerous courses of antibiotics. To assess antibody-mediated immunity, we evaluated their responses to tetanus and diphtheria vaccination and compared that to the responses in healthy individuals.

\section{MATERIALS AND METHODS}

Study participants 
The study patients were selected from 198 adult patients who were admitted between 1995 and 1997 to Jorvi Hospital, Espoo, Finland because of recurrent or chronic rhinosinusitis, and who were evaluated and operated on by one of the authors (O.T.). Inclusion criteria for patients with chronic rhinosinusitis included continuous symptoms of sinus inflammation manifesting as continuously stuffed nose with nasal discharge for more than two years with periodic exacerbations of purulent sinusitis. Inclusion criteria for patients with recurrent rhinosinusitis were more than four episodes of acute sinusitis during the last 12 months or more than two episodes of acute sinusitis a year during the last five years. Between the acute sinusitis episodes the patients were relatively free of sinonasal symptoms. Diagnosis of acute sinusitis was defined when effusion was detected in maxillary sinuses by maxillary sinus punctures or sinus roentgenograms. Patients that had received peroral corticosteroid therapy within the last 12 months were excluded from the study.

Of the 25 patients included in the study, 13 patients presented with chronic and 12 patients presented with recurrent rhinosinusitis. The mean age of the patients was 46 years (range 19 to 64 years). Eighteen were female and 7 were male. All the patients underwent endoscopic sinus surgery. The mean preoperative duration of symptoms related to recurrent or chronic sinusitis was 7 years (range 1 to 22 years). Nine of the patients had asthma. Four patients had specific serum IgE antibodies to pollen or animal dust antigens. Nasal polyposis was present in 15 patients. All the patients had normal levels of serum IgA, $\mathrm{IgM}, \mathrm{IgG}$, as well as normal levels of IgG subclasses.

Thirteen study patients experienced one sinus operation and the rest of them from 2 to 5 operations. Two of the study patients were operated on endoscopically by a modified Lothrop procedure to drain the frontal sinuses. The study patients represented a subpopulation having the most severe spectrum of sinusitis symptoms of the patients admitted to Jorvi hospital.

Healthy individuals with no prior history of recurrent of chronic sinusitis were recruited among hospital personnel. Healthy individuals were selected to match the sex and age distribution of the patients. Altogether, 30 control individuals were recruited. Twenty-six were females and 4 were males. The mean age was 43 years (range 25-61 years).

The Ethical Review Committee of the Helsinki University Central Hospital had approved the study. All the study subjects gave written informed consent to participate in the study. The study was conducted in accordance with the regulatory requirements, Good Clinical Practice, and the ethical principles of the Declaration of Helsinki.

\section{Vaccination and serum samples}

All the study participants were vaccinated with tetanus and diphtheria toxin vaccine. The vaccine, Tetanus-d-rokote ${ }^{\circledR}$, was manufactured at the National Public Health Institute, Helsinki. It contained $2 \mathrm{Lf}$ diphtheria and $5 \mathrm{Lf}$ tetanus toxoid absorbed to aluminum phosphate in $0.5 \mathrm{ml}{ }^{(21)}$. It was administered by an intramuscular injection once into the upper deltoid. Vaccination of the patients was performed when the patients did not have any acute exacerbations of their sinus disease. Peripheral blood for serum samples was taken immediately before vaccination and 2 weeks after. Serum samples were stored frozen until analysis.

\section{Determination of serum antibody concentrations}

Diphtheria and tetanus antitoxins were measured by a double antigen EIA (DAE) as described previously (21). The antibody concentrations were reported as international units per $\mathrm{mL}$ (IU/mL). Tetanus and diphtheria antitoxin concentrations between $0.01-0.1 \mathrm{IU} / \mathrm{mL}$ are conventionally considered as low positive, whereas antitoxin concentrations $\geq 0.1 \mathrm{IU} / \mathrm{mL}$ are considered positive ${ }^{(22)}$. Concentrations $\geq 1 \mathrm{IU} / \mathrm{mL}$ indicate a good long-term protection.

\section{Statistical analysis}

Statistical analyses of antibody concentrations were performed after logarithmic transformation of the data. Linear regression was used to compare antibody responses. Fisher exact test was used to calculate the statistical significance of two-way tables. Logistic regression was used to analyze possible parameters associated with a defective antibody response. The analyses were performed using the PASW Statistics 18.0 software (SPSS Inc., Chicago, IL, USA).

\section{RESULTS}

At the start of the trial most study participants had tetanus and diphtheria toxoid antibodies. This reflected previous vaccinations against tetanus and diphtheria. However, some had very low tetanus ( 2 patients) or diphtheria (1 patient and 4 controls) antitoxin concentrations $(<0.01 \mathrm{IU} / \mathrm{mL})$ before the vaccination. This might indicate that these individuals may not have previously been vaccinated or have received incomplete primary vaccinations. As the primary and secondary antibody responses are likely very different, we excluded these individuals from the analysis.

Vaccination induced strong antibody responses 2 weeks after vaccination. Among the patients, diphtheria toxoid induced on the average a 5.74-fold rise (95\% CI 3.54 to 9.29$)$ and tetanus toxoid induced on the average a 3.48 -fold rise (95\% CI 2.19 to 5.51) in antibody concentrations. However, these responses were higher among the healthy controls, a 12.9 -fold rise $(95 \%$ CI 8.13 to 20.4 ) and a 6.93 -fold rise ( $95 \%$ CI 4.38 to 11.0 ), respectively. When antibody responses were compared between patients and controls, we found lower responses in patients as compared to healthy controls. Diphtheria toxoid induced on the average 4.08-fold lower responses and tetanus toxoid induced on the average 2.20-fold lower responses in patients with rhinosinusitis than in healthy controls (Table 1). Before the vaccination, we could not observe any significant differences in antibody concentrations between the healthy controls and the patients (Table 1). 
Confounding factors, particularly the antibody level before the vaccination may have caused a bias. To test this, we did linear regression where the pre-vaccination antibody level, gender, and age, as well as the study subject group (patient vs. control), were entered as independent variables and the post-vaccination antibody level was entered as the dependent variable. The analysis revealed that after correcting for the above confounding factors the patients had on average 2.72-fold lower (95\% CI 1.51 to $4.89, \mathrm{p}=0.001)$ and 2.33 -fold lower $(95 \% \mathrm{CI}$ 1.64 to $3.31, \mathrm{p}<0.001)$ antitoxoid responses to diphtheria and tetanus, respectively. This implied that the patients had low responses irrespective of age, gender, or the pre-vaccination antibody level.

To approximate a normal antibody response to diphtheria and tetanus vaccination, we plotted the post-vaccination antibody concentrations in a graph (Figure 1). The antibody responses in patients were variable in that some patients had responses comparable to that of the healthy controls but some had clearly lower responses than the healthy controls. Such low responders did not develop high enough antibody concentrations that would reach the $95 \%$ normal distribution range of healthy controls. Eleven (46\%) out of 24 patients (p $<0.001)$ and $7(30 \%)$ out of 23 patients $(\mathrm{p}=0.02)$ were such low responders to diphtheria and tetanus toxoids, respectively (Figure 1). Out of 25 patients, $14(56 \%)$ were low responders to either diphtheria or tetanus $(\mathrm{p}<0.001)$ and $4(16 \%)$ patients had low responses to both diphtheria and tetanus.

We then evaluated whether an abnormal antibody response would correlate with any clinical symptoms or findings. Although the number of patients who presented with recurrent as opposed to chronic rhinosinusitis was somewhat higher among the low responders, the difference was not statistically significant (Table 2). We could not observe any significant association between vaccine responses and polyposis, asthma, or the presence of serum IgE antibodies to pollen or animal dust antigens (Table 2). There were no statistically significant differences in serum immunoglobulin concentrations between defective vs. normal responders (data not shown).

\section{DISCUSSION}

Comparison of the responses after vaccination revealed that antibody responses to tetanus or diphtheria toxoids were low in approximately half of our rhinosinusitis patients. This may indicate a generalized impairment of antibody responses in a significant proportion of rhinosinusitis patients that may predispose them for the disease. Alternatively, it is possible that a chronic inflammation in the nasal sinuses may secondarily impair systemic antibody responses. This could further impair the ability to recover from sinusitis.

We excluded patients who had been using oral steroids within the last 12 months. Despite this, many of our patients were users of inhaled steroids. This could have impaired vaccine responses. However, our patients were free of acute symptoms

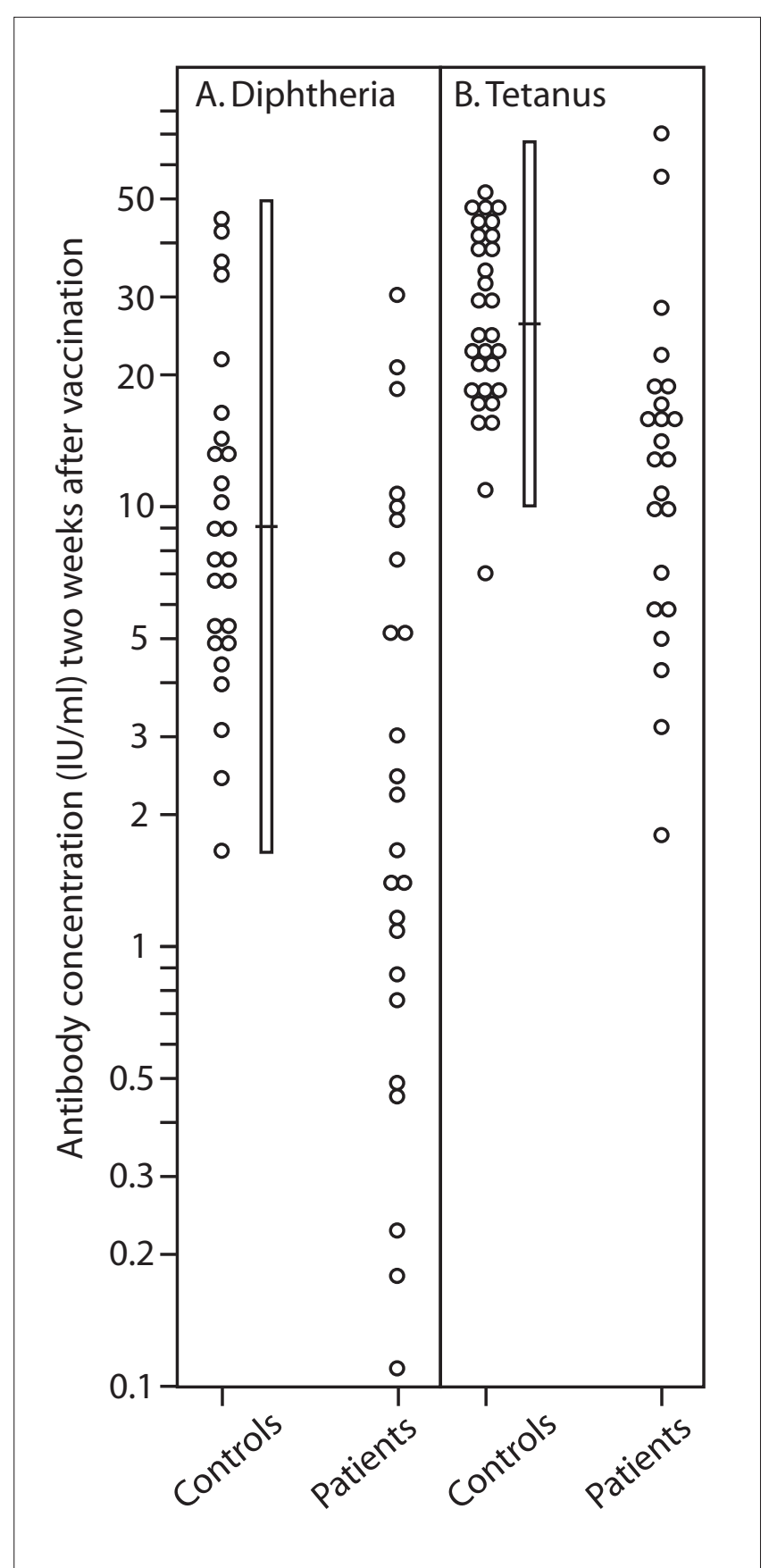

Figure 1. Antibody responses 2 weeks after diphtheria and tetanus toxoid vaccination. A. Diphtheria toxoid antibody concentrations in healthy control individuals and in CRS patients. B. Tetanus toxoid antibody concentrations in healthy control individuals and in CRS patients. Each antibody value is indicated by a circle. The $95 \%$ normal distribution of antibody concentrations and the geometric mean antibody concentration in healthy control individuals is indicated by a box and a line, respectively.

at the time of the vaccination and the use for inhaled steroids may have not been very excessive at that time. This suggests that the use of inhaled steroids are likely to not have introduced a strong bias to our results.

It should be emphasized that the antibody responses measured represented secondary responses, as tetanus and diphtheria vaccination has been included in the Finnish vaccination pro- 
Table 1. Comparison of antibody responses to vaccination between rhinosinusitis patients and controls.

\begin{tabular}{|c|c|c|c|c|}
\hline & Patients & Controls & & \\
\hline & $\begin{array}{l}\text { Geometic mean } \\
\text { (range) in IU/mL }\end{array}$ & $\begin{array}{l}\text { Geometic mean } \\
\text { (range) in IU/mL }\end{array}$ & $\begin{array}{c}\text { Ratio of the geometric means }(95 \% \mathrm{CI}) \\
\text { between controls and patients }\end{array}$ & $\mathrm{p}$-value \\
\hline Diphtheria toxoid antibodies & $\mathrm{n}=24$ & $\mathrm{n}=26$ & & \\
\hline before vaccination & $0.38(0.04-9.1)$ & $0.70(0.06-6.4)$ & $1.82(0.83-3.97)$ & 0.13 \\
\hline 2 weeks after vaccination & $2.22(0.1-31)$ & $9.08(1.6-45)$ & $4.08(2.00-8.36)$ & $<0.001$ \\
\hline Tetanus toxoid antibodies & $\mathrm{n}=23$ & $\mathrm{n}=30$ & & \\
\hline before vaccination & $3.40(0.3-12.9)$ & $3.75(0.1-24)$ & $1.10(0.59-2.04)$ & 0.75 \\
\hline 2 weeks after vaccination & $11.8(1.8-71)$ & $25.9(7.0-51)$ & $2.20(1.51-3.19)$ & $<0.001$ \\
\hline
\end{tabular}

Table 2. Correlation of co-morbidities with a defective antibody response. The values are numbers of patients unless otherwise stated.

\begin{tabular}{|c|c|c|c|c|c|}
\hline & \multirow{3}{*}{$\begin{array}{l}\text { Normal response } \\
n=11\end{array}$} & \multicolumn{4}{|c|}{ Defective response } \\
\hline & & \multicolumn{2}{|c|}{ Either diphtheria or tetanus } & \multicolumn{2}{|c|}{ Both diphtheria and tetanus } \\
\hline & & $\mathrm{n}=14$ & OR $(95 \% \mathrm{CI})$ & $\mathrm{n}=4$ & OR $(95 \% \mathrm{CI})$ \\
\hline Recurrent rhinosinusitis & $4(36 \%)$ & $8(57 \%)$ & $2.33(0.46$ to 11.81$)$ & $2(50 \%)$ & $1.75(0.17$ to 17.68$)$ \\
\hline Polyposis & $7(63 \%)$ & $8(57 \%)$ & $0.76(0.15$ to 3.87$)$ & $2(50 \%)$ & $0.57(0.06$ to 5.78$)$ \\
\hline Asthma & $3(27 \%)$ & $6(43 \%)$ & $2.00(0.37$ to 10.92$)$ & $3(75 \%)$ & $8.00(0.58$ to 110.27$)$ \\
\hline $\begin{array}{l}\text { Serum IgE antibodies to } \\
\text { pollen or animal dust }\end{array}$ & $2(18 \%)$ & $2(14 \%)$ & $0.75(0.09$ to 6.39$)$ & $0(0 \%)$ & - \\
\hline
\end{tabular}

gram of children already for 50 years. In addition, our study individuals had already tetanus and diphtheria antibodies at the start of the trial and those who had extremely low levels of antibodies were excluded from the analysis. Thus, patients with rhinosinusitis may have impairments in secondary antibody responses.

Before vaccination, we could not observe any statistically significant differences between the patients and controls in tetanus and diphtheria antibody concentrations. The average pre-vaccination antibody concentrations were very similar in patients and controls. After including the pre-vaccination antibody level in the linear regression analyses, the patients had still on the average lower antibody responses than the controls. This supports the conclusion that a subset of our patients had an impaired antibody response to vaccination.

We made an attempt to analyze the phenotype of patients who had impairments in antibody responses. Our analysis showed that the patient may have an impaired antibody response regardless of the presence of polyposis and irrespective if the patient had chronic or recurrent symptoms of sinusitis. Patients with recurrent sinusitis tended to have more frequently defective vaccine responses, but our study was too small to address if this was real.

The study patients represented patients who had especially severe symptoms of chronic or recurrent sinusitis despite endoscopic surgery. As the severity and type of symptoms are rather variable among rhinosinusitis patients, it is possible that the true prevalence of impaired antibody mediated immu- nity in patients who initially present with chronic or recurrent rhinosinusitis is much lower than observed in our study and are patients who may represent the most extreme spectrum of disease severity. Nevertheless, our study shows than when the clinician faces a patient with persisting or recurrent symptoms of sinusitis, the patient may suffer from an impairment of antibody-mediated immunity.

Impaired responses to vaccination to polysaccharide antigens have previously been observed in CRS suggesting that a subset of patients has impairments of antibody-mediated immunity ${ }^{117-}$ ${ }^{20)}$. Some of these patients may benefit from intravenous immunoglobulin therapy ${ }^{(19)}$. Our results imply that, in addition to polysaccharide vaccines, vaccine responses to protein antigens can be used in the evaluation of antibody-mediated immunity of rhinosinisitis patients.

Antibody responses to protein as compared to polysaccharide antigens are distinct as they are different in the mechanisms how antigen presenting cells present the antigen to B lymphocytes as well as different in their potency to elicit secondary responses ${ }^{(23)}$. Thereby, concurrent evaluation of both protein and polysaccharide vaccine responses may give more information of immune abnormalities among patients with recurrent or chronic infections.

\section{REFERENCES}

1. Knight AK, Cunningham-Rundles C. Inflammatory and autoimmune complications of common variable immune deficiency. Autoimmun Rev. 2006; 5: 156-159.

2. Ogershok PR, Hogan MB, Welch JE, et al. Spectrum of illness in pediatric common variable immunodeficiency. Ann Allergy 
Asthma Immunol. 2006; 97: 653-656.

3. Oksenhendler E, Gerard L, Fieschi C, et al. Infections in 252 patients with common variable immunodeficiency. Clin Infect Dis. 2008; 46: 1547-1554.

4. Plebani A, Soresina A, Rondelli R, et al. Clinical, immunological, and molecular analysis in a large cohort of patients with X-linked agammaglobulinemia: an Italian multicenter study. Clin Immunol. 2002; 104: 221-230.

5. Winkelstein JA, Marino MC, Ochs H, et al. The X-linked hyperIgM syndrome: clinical and immunologic features of 79 patients. Medicine 2003; 82: 373-384.

6. Jackson LL, Kountakis SE. Classification and management of rhinosinusitis and its complications. Otolaryngol Clin North Am. 2005; 38: 1143-1153.

7. Shapiro GG, Virant FS, Furukawa CT, et al. Immunologic defects in patients with refractory sinusitis. Pediatrics 1991; 87: 311-316.

8. Wise MT, Hagaman DD. An immunological approach to chronic and recurrent sinusitis. Curr Opin Otolaryngol Head Neck Surg. 2007; 15: 10-17.

9. Lund VJ, Scadding GK. Immunologic aspects of chronic sinusitis. J Otolaryngol. 1991; 20: 379-381.

10. Scadding GK, Lund VJ, Darby YC, et al. IgG subclass levels in chronic rhinosinusitis. Rhinology 1994; 32: 15-19.

11. Armenaka M, Grizzanti J, Rosenstreich DL. Serum immunoglobulins and IgG subclass levels in adults with chronic sinusitis: evidence for decreased IgG3 levels. Ann Allergy 1994; 72: 507-514.

12. Aghamohammadi A, Mohammadi J, Parvaneh N, et al. Progression of selective IgA deficiency to common variable immunodeficiency. Int Arch Allergy Immunol. 2008; 147: 87-92.

13. Janzi M, Kull I, Sjöberg R, et al. Selective IgA deficiency in early life: association to infections and allergic diseases during childhood. Clin Immunol. 2009; 133: 78-85.

14. Costa Carvalho BT, Nagao AT, Arslanian C, et al. Immunological evaluation of allergic respiratory children with recurrent sinusitis. Pediatr Allergy Immunol. 2005; 16: 534-538.

15. Vanlerberghe L, Joniau S, Jorissen $\mathrm{M}$. The prevalence of humoral immunodeficiency in refractory rhinosinusitis: a retrospective analysis. B-ENT. 2006; 2: 161-166.

16. Tahkokallio O, Seppälä IJ, Sarvas H, et al. Concentrations of serum immunoglobulins and antibodies to pneumococcal capsular polysaccharides in patients with recurrent or chronic sinusitis. Ann Otol Rhinol Laryngol. 2001; 110: 675-681.

17. Silk H, Zora J, Goldstein J, et al. Response to pneumococcal immunization in children with and without recurrent infections. J Asthma 1998; 35: 101-112.
18. Epstein MM, Gruskay F. Selective deficiency in pneumococcal antibody response in children with recurrent infections. Ann Allergy Asthma Immunol. 1995; 75: 125-131.

19. Sethi DS, Winkelstein JA, Lederman H, et al. Immunologic defects in patients with chronic recurrent sinusitis: diagnosis and management. Otolaryngol Head Neck Surg. 1995; 112: 242-247.

20. May A, Zielen S, von Ilberg C, et al. Immunoglobulin deficiency and determination of pneumococcal antibody titers in patients with therapy-refractory recurrent rhinosinusitis. Eur Arch Otorhinolaryngol. 1999; 256: 445-449.

21. Ölander RM, Wuorimaa T, Käyhty H, et al. Booster response to the tetanus and diphtheria toxoid carriers of 11-valent pneumococcal conjugate vaccine in adults and toddlers. Vaccine 2001;20: 336-341.

22. Ramsay ME, Rao M, Begg NT, et al. Antibody response to accelerated immunisation with diphtheria, tetanus, pertussis vaccine. Lancet 1993; 342: 203-205.

23. Gonzalez-Fernandez A, Faro J, Fernandez C. Immune responses to polysaccharides: lessons from humans and mice. Vaccine 2008; 26: $292-300$.
Petri S Mattila

Department of Otorhinolaryngology

Helsinki University Central Hospital

Haartmaninkatu 4 E

P.O. Box 220

00290 Helsinki

Finland

Tel: +358-50-427 1560

E-mail: petri.mattila@hus.fi 